\title{
Caracterización de donadores seropositivos por Trypanosoma cruzi y su manejo, Banco de Sangre, Hospital Escuela, Tegucigalpa, 2016
}

\author{
Characterization and management of Trypanosoma cruzi seropositive donors, \\ Blood Bank, Hospital Escuela, Tegucigalpa, 2016 \\ José Orlando Maldonado Triminio \\ Médico Especialista en Medicina Interna; Hospital de Especialidades San Felipe; \\ Instituto Nacional Cardiopulmonar; Tegucigalpa.
}

\begin{abstract}
RESUMEN. Antecedentes: La eliminación de la transmisión no-vectorial de Trypanosoma cruzi es objetivo nacional de salud pública. La seroprevalencia en donadores de sangre es blanco de estrategias de eliminación de la Enfermedad de Chagas. Objetivo: Caracterizar donadores seropositivos por T. cruzi y su manejo en el Banco de Sangre, Hospital Escuela, Tegucigalpa, 2016. Metodología: Estudio descriptivo transversal sobre muestra aleatoria de 150 donadores de sangre, mayores de 18 años de edad, seleccionados a partir de un sub-grupo de 726 casos seropositivos por enfermedades de notificación obligatoria. Los donadores seropositivos por T. cruzi fueron invitados a participar mediante seguimiento telefónico. Se registró información sobre diagnóstico, características sociodemográficas, notificación, referencia y seguimiento. El protocolo fue aprobado por comité de ética institucional, Facultad de Ciencias Médicas UNAH. Resultados: Se identificaron 30 casos seropositivos por T. cruzi (20.0\%, IC95\% 13.3-26.7), edad media 39.1 años, sexo masculino $66.7 \%$ (20), procedentes de regiones centro-sur-oriente y de bajo nivel socioeconómico. De estos 30 casos, 15 respondieron y aceptaron participar; de estos, 6 informaron que se les notificó el resultado serológico y 5 fueron referidos para evaluación y tratamiento. Entre estos 5, en un caso se realizó una segunda prueba diagnóstica. Ninguno fue notificado sobre su aptitud para donar sangre. Conclusión: Los casos identificados son adultos jóvenes, con relación hombre a mujer 2:1. Es necesario implementar un sistema de registro nacional de notificación y seguimiento de casos identificados en los bancos de sangre. Este sistema debe estar integrado al sistema de vigilancia de la Secretaría de Salud de Honduras.
\end{abstract}

Palabras clave: Enfermedad de Chagas, Medicina Transfusional, Transfusión de componentes sanguíneos, Trypanosoma cruzi.

\section{INTRODUCCIÓN}

La evolución y avances de la medicina transfusional, así como el impacto de ésta sobre el pronóstico del paciente, la han convertido en una de las herramientas más importantes de la medicina moderna; aunque no exenta de efectos adversos. Entre estos está el de la infección transmitida a través de productos sanguíneos, como es el caso de la transmisión de la Enfermedad de Chagas, cuyo agente etiológico es el protozoo Trypanosoma cruzi. Originalmente sospechada en 1936 por Mazza et al, Dias y Pellegrino en 1949, fue demostrada por Freitas et al en 1950 en bancos de sangre de Sao Paulo, Brasil. ${ }^{1}$ En 1972 se identificó la asociación entre número de transfusiones y seroprevalencia por $T$. cruzi en pacientes hemofílicos, con prevalencia efectiva de transmisión en $12.5-25.0 \%$ de receptores de sangre contaminada con T. cruzi. ${ }^{1}$ En 2009 se estimó una seroprevalencia por T. cruzi en 17 millones de latinoamericanos, de los cuales la mitad con enfermedad latente ${ }^{2}$ y 100 millones en riesgo de contagio, $60 \%$ de ellos viviendo en zonas urbanas. ${ }^{3}$

Recibido 30-1-2019; Aceptado para publicación 15-3-2019.

Dirección para correspondencia: José Orlando Maldonado Triminio

Correo electrónico: maldonadotriminio@gmail.com

Conflictos de interés. El autor declara no poseer conflictos de interés en relación a este artículo.

DOl: https://doi.org/10.5377//rmh.v87i1.11926
Existe una amplia variación regional, pasando en algunas zonas de Bolivia de $46.5 \%$ a México que reporta tasas general de $1.6 \%$ y de $4.5-5 \%$ en otras. ${ }^{1,4,5}$

A pesar de los avances en la prevención de la infección, en países de altos ingresos se informa contaminación en 1/5,000 transfusiones plaquetarias o 1/30,000 transfusiones sanguíneas. ${ }^{6-9}$ Aún en Estados Unidos de América con seroprevalencia poblacional de $0.1-0.2 \%$ se informó 7 casos positivos en $2014 .^{8-}$ ${ }^{10}$ El riesgo existe y depende de: a) Prevalencia de infección en donadores, b) Nivel de cobertura serológica, c) Sensibilidad de las pruebas utilizadas, d) Seguridad de los resultados obtenidos, e) Riesgo., ${ }^{2,11} \mathrm{Al}$ final de la década de los años setenta Brasil informaba 10,000 nuevos casos anuales de Enfermedad de Chagas asociados a transfusión de productos sanguíneos, país que inició la implementación del tamizaje de laboratorio por $T$. cruzi así como la aplicación de los estándares actuales aplicados en Latinoamérica. Brasil logró a finales de la década de los noventa reducir la incidencia a 30 casos anuales y logró la eliminación de la transmisión por transfusión de T. cruzi en 2006, aunque primero lo lograron Uruguay y Chile en 1997 y 1999 , respectivamente. ${ }^{3}$ No obstante, el éxito aún no es total en la región, en 2009 se había logrado disminución de $70 \%$ en la incidencia de casos por esta vía. ${ }^{3}$

La reinfestación o reinvasión es parte importante de esta problemática de salud pública, por lo que la Organización 
Panamericana de la Salud/Organización Mundial de la Salud implementó la certificación de cumplimiento de "interrupción de la vía de transmisión de T. cruzi" por vías no vectoriales en países de Latinoamérica, región en la que también impulsa la "Iniciativa del Cono Sur" para prevención de transmisión de $T$. cruzi desde 1991. Esta iniciativa incluye pautas y reglamentos para el manejo de los productos sanguíneos, así como protocolos de protección., ${ }^{2,3}$ La piedra angular de estas estrategias está conformada por tamizaje epidemiológico mediante aplicación de un cuestionario que incluye antecedentes y factores de riesgo así como detección por pruebas de laboratorio, los Bancos de Sangre deben mantener una lista de donadores proscritos, así como la implementación de un sistema de notificación y seguimiento epidemiológico en cada Banco de Sangre a nivel regional. ${ }^{7,9,12}$

En Honduras se ha eliminado la transmisión de T. cruzi por el vector Rodnius prolixus, con importantes avances en el control de Triatoma dimidiata, con un informe en 2012 de 98 casos nuevos a nivel nacional de Enfermedad de Chagas. ${ }^{13} \mathrm{La}$ seroprevalencia poblacional pasó de $36.8 \%$ en 1973 a $2.6 \%$ en 2011; y en donadores en bancos de sangre nacionales pasó de $28 \%$ a $1.5 \%$ en 2009 , de acuerdo a lo informado por la Secretaría de Salud de Honduras. ${ }^{14-16}$ La detección, monitoreo y seguimiento terapéutico de casos no diagnosticados e identificados en donadores de sangre es parte de las estrategias de eliminación no vectorial de la Enfermedad de Chagas en Honduras. Sin embargo, no se cuenta con este tipo de información en el Banco de Sangre del Hospital Escuela, el principal centro hospitalario de atención pública en el país, siendo este el banco de sangre con mayor volumen de transfusiones a nivel nacional por lo que se consideró importante generar información sobre esta problemática de salud pública. Este estudio se realizó con el objetivo de caracterizar donadores seropositivos por T. cruzi y su manejo en el Banco de Sangre del Hospital Escuela, Tegucigalpa, en el año 2016.

\section{METODOLOGIA}

Estudio descriptivo transversal sobre donadores de sangre mayores de 18 años seropositivos por $T$. cruzi identificados en el Banco de Sangre del Hospital Escuela, Tegucigalpa, en el año 2016. Los casos se identificaron a partir de una muestra aleatoria de 150 casos de un sub-grupo de 726 casos seropositivos por enfermedades transmisibles de notificación obligatoria en Bancos de Sangre incluyendo Enfermedad de Chagas, HTLV-I y II, sífilis y VIH, utilizando los parámetros proporción esperada de casos positivos $50 \%$, precisión de $5.0 \%$ y poder estadístico de $80 \%$. El estudio se realizó en dos etapas.

En la primera etapa, se recopilaron los formularios de los 150 donadores seropositivos por enfermedades transmisibles de notificación obligatoria en Bancos de Sangre para identificar entre ellos los casos seropositivos por $T$. cruzi. La técnica empleada en el Banco de Sangre del Hospital Escuela para identificar casos es ELISA (Enzyme-Linked Inmunosorbent Assay) para $T$. cruzi, método que se basa en la detección de inmu- noglobulinas IgG anti $T$. cruzi en sangre de los pacientes y su reacción colorimétrica visible en el caso de que la sangre del paciente contenga los anticuerpos. Para los casos seropositivos por $T$. cruzi se registró la información en un instrumento diseñado y validado para tal efecto, compuesto por secciones para registrar datos generales, información sobre exposición y riesgo de infección y antecedentes personales.

La segunda etapa consistió en seguimiento telefónico de los casos identificados seropositivos por $T$. cruzi. Los casos contactados fueron invitados a participar en el estudio mediante consentimiento informado. Los casos que aceptaron participar fueron interrogados y la información se registró en un segundo instrumento incluyendo información general y relacionada con la notificación, referencia y seguimiento de la Enfermedad de Chagas por parte del Banco de Sangre del Hospital Escuela.

La información recopilada fue digitada en una base de datos electrónica generada con Epi-Info 7 (CDC, Atlanta, EUA, 2015), programa con el que se generaron listados de frecuencias y cruce de variables. Se determinaron intervalos de confianza de 95\% (IC95\%, método binomial, EpiTable 1.0, Epi-Info 6.04d, CDC, Atlanta, EUA, 2001).

El estudio fue aprobado por el Comité de Ética en Investigación Biomédica (CEIB) de la Facultad de Ciencias Médicas UNAH. A los participantes contactados por teléfono se les explicó el objetivo del estudio y la finalidad de contribuir a la mejora en la atención brindada en el Hospital Escuela. A cada participante se le proporcionó información sobre los resultados de los exámenes de laboratorio practicados en el Banco de Sangre y se les facilitó su referencia al Centro de Atención Integral (CAI) del Hospital Escuela, para manejo en caso de ser necesario.

\section{RESULTADOS}

A partir de la muestra aleatoria de 150 donadores de los 726 seropositivos por enfermedades transmisibles de notificación obligatoria en Bancos de Sangre, se identificaron $30 \mathrm{ca}-$ sos seropositivos por T. cruzi para una proporción de $20.0 \%$ (IC95\%13.9-27.3). En la Figura 1 se describe el diagrama de flujo del estudio a partir de estos 30 donadores, cuyas características sociodemográficas se presentan en el Cuadro 1. Se estimó una edad media de 39.1 años, siendo el grupo de edad más frecuente el grupo 28-37 años con 43.3\% (13). La mayoría pertenecía al sexo masculino $66.7 \%$ (20), con una relación sexo masculino a femenino de 2:1. La mayoría procedía de Francisco Morazán y El Paraíso, 23.3\% (7) cada uno. Las ocupaciones más frecuentes fueron agricultor $30.0 \%$ (9) y comerciante $20.0 \%$ (6). Según el tipo de sangre y Rh, fueron O Positivo $66.7 \%$ (20), A Positivo $23.3 \%$ (7). Eran donadores por primera vez $36.7 \%$ (11).

De los 30 donadores seropositivos contactados telefónicamente, 15 respondieron la llamada y todos aceptaron participar en el estudio. De estos 15, 6 respondieron que habían sido informados sobre el resultado seropositivo por $T$. cruzi y 5 informaron que fueron referidos al Centro de Atención Integral (CAI) del Hospital Escuela para tratamiento. En el Cuadro 2 se 
describen algunas características sociodemográficas y sobre la donación. Todos estos 5 donadores recibieron tratamiento y de éstos, cuatro estaban en tratamiento cuando fueron contactados; dos fueron citados para evaluación y dos fueron citados dos veces. Solamente un caso informó que le fue realizado una segunda prueba de laboratorio. Ninguno de estos cinco donadores fue informado sobre su aptitud para donar sangre. A los 9 donadores seropositivos que refirieron no haber recibido notificaciones y/o seguimiento, se les proporcionó consejería telefónica y todos fueron referidos al Centro de Atención Integral (CAl) del Hospital Escuela (Ver Figura 1).

\section{DISCUSIÓN}

En este estudio se encontró que la mayoría de los donadores seropositivos no fueron notificados sobre su condición, así tampoco referidos para tratamiento, sino hasta el momento en que fueron contactados vía telefónica por el investigador. Estos resultados reflejan debilidades en el sistema de notificación y seguimiento de casos seropositivos por parte del Banco de Sangre del Hospital Escuela. En general los bancos de sangre juegan un papel como sensores epidemiológicos al realizar los análisis serológicos para la detección de enfermedades transmisibles como ser la Enfermedad de Chagas. Estas enfermedades son consideradas como problemas de salud pública y de acuerdo con la normativa son objeto de notificación obligatoria, por lo que se debe establecer una red de comunicación y diagnóstico para el seguimiento de los casos. ${ }^{12}$

El perfil sociodemográfico del donador seropositivo por $T$. cruzi en el Banco de Sangre del Hospital Escuela se correspondió con individuos en la cuarta década de la vida, de sexo masculino en más de dos tercios de la muestra estudiada, duplicando en frecuencia al sexo femenino. La mayor parte procedente de las regiones central, sur y oriental del país, predominando oficios como agricultor, comerciante y ama de casa, residente en ambiente urbano ó urbano-marginal en cuatro quintas partes (Cuadro 1). Dentro de las características del donador hay una que es objetivo de los programas nacionales e internacionales para el aumento en la seguridad transfusional, que es lograr que $100 \%$ de los donadores sean voluntarios espontáneos no

Figura 1. Diagrama de flujo del estudio, donadores seropositivos por Trypanosoma cruzi y su manejo, Banco de Sangre, Hospital Escuela, Tegucigalpa, 2016.

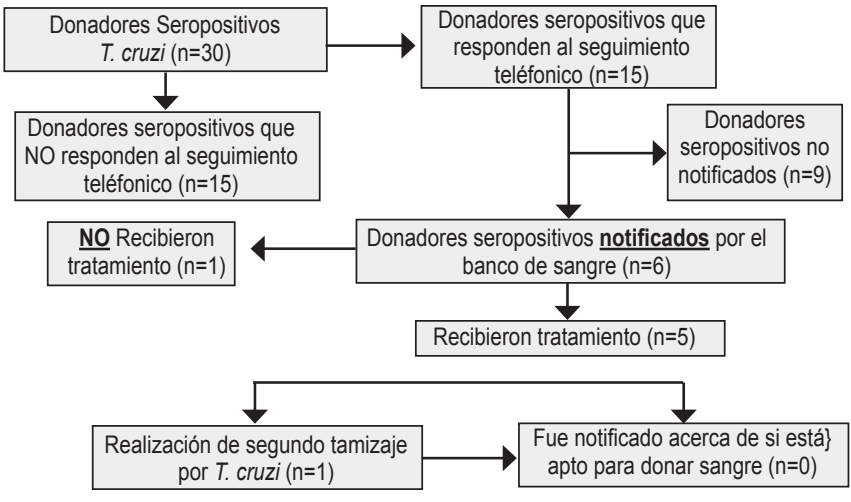

remunerados, ya que se ha identificado que es el tipo de sangre más segura. ${ }^{7,17}$ En este estudio el tipo de donador fue familiar o allegado $86.7 \%$; lo que no cumple el objetivo de lograr el $100 \%$ de donación voluntaria como medio para obtener sangre totalmente segura en Honduras. Casi la totalidad de lo captado en Bancos de Sangre procede de donadores de reemplazo, es decir que un paciente que será sometido a una cirugía debe proporcionar dos o más donantes, usualmente familiares o allegados; sin embargo, para cambiar esta situación y lograr sangre $100 \%$ segura, es necesario renovar el marco legislativo actual añadiendo aspectos relacionados con la obtención de esta meta, que no está incluida en la legislación actual. ${ }^{13,18-20}$

La proporción estimada de seropositividad por $T$. cruzi en este estudio, en esta muestra aleatoria de un subgrupo de seropositivos por enfermedades de notificación obligatoria, fue $20.0 \%$ (IC95\% (IC95\% 13.9-27.3). Por la falta de registros disponibles en el Banco de Sangre del Hospital Escuela y el tipo de muestreo utilizado en este estudio, no fue posible estimar la seroprevalencia por $T$. cruzi, solo una proporción entre un grupo ya seleccionado como seropositivo. Para el año 2012, en un

Cuadro 1. Características sociodemográficas de los pacientes, donadores seropositivos por Enfermedad de Chagas, Banco de Sangre, Hospital Escuela, Tegucigalpa, 2016, $n=30$.

\begin{tabular}{|c|c|c|}
\hline Características & $\mathbf{N}$ & $(\%)$ \\
\hline $\begin{array}{l}\text { Edad (años) } \\
18-27 \\
28-37 \\
38-47 \\
48-57 \\
\text { Media } 39.1 \\
\text { DS } \pm 8.8 \\
\text { Rango 18-56 }\end{array}$ & $\begin{array}{c}2 \\
13 \\
10 \\
5\end{array}$ & $\begin{array}{c}(6.7) \\
(43.3) \\
(33.3) \\
(16.7)\end{array}$ \\
\hline $\begin{array}{l}\text { Sexo } \\
\text { Masculino } \\
\text { Femenino }\end{array}$ & $\begin{array}{l}20 \\
10\end{array}$ & $\begin{array}{l}(66.7) \\
(33.3)\end{array}$ \\
\hline $\begin{array}{l}\text { Procedencia } \\
\text { Francisco Morazán } \\
\text { El Paraíso } \\
\text { Olancho } \\
\text { Intibucá } \\
\text { Comayagua } \\
\text { La Paz } \\
\text { Cortés } \\
\text { Santa Bárbara } \\
\text { Lempira } \\
\text { Valle }\end{array}$ & $\begin{array}{l}7 \\
7 \\
3 \\
3 \\
2 \\
2 \\
2 \\
2 \\
1 \\
1\end{array}$ & $\begin{array}{l}(23.3) \\
(23.3) \\
(10.0) \\
(10.0) \\
(6.7) \\
(6.7) \\
(6.7) \\
(6.7) \\
(3.3) \\
(3.3)\end{array}$ \\
\hline $\begin{array}{l}\text { Ocupación } \\
\text { Agricultor } \\
\text { Comerciante } \\
\text { Ama de casa } \\
\text { Motorista } \\
\text { Guardia de seguridad } \\
\text { Albañil } \\
\text { Militar } \\
\text { Oficinista } \\
\text { Técnico en mantenimiento } \\
\text { Fotógrafo } \\
\text { Estudiante }\end{array}$ & $\begin{array}{l}9 \\
6 \\
4 \\
2 \\
2 \\
2 \\
1 \\
1 \\
1 \\
1 \\
1\end{array}$ & $\begin{array}{l}(30.0) \\
(20.0) \\
(13.3) \\
(6.7) \\
(6.7) \\
(6.7) \\
(3.3) \\
(3.3) \\
(3.3) \\
(3.3) \\
(3.3)\end{array}$ \\
\hline
\end{tabular}

DS= desviación estándar 
informe del Programa Nacional de Prevención y Control de la Enfermedad de Chagas de la Secretaría de Salud de Honduras, ${ }^{15}$ que cumplía funciones de regulación, monitoreo y control de estándares de los bancos de sangre del país, incluso los de organizaciones como Cruz Roja Internacional y hospitales privados, se reportó una seroprevalencia del $1.5 \%$. Aunque el Hospital Escuela es el hospital público terciario más importante a nivel nacional y su banco de sangre es el de mayor demanda en el país, la cobertura de atención de pacientes procedentes del norte y nor-occidente es reducida.

La implementación de estrategias de notificación y seguimiento del donador seropositivo para ser referido a tratamiento y posterior monitoreo del resultado terapéutico es parte esencial de un sistema de manejo de donadores en Bancos de Sangre y en concordancia con los lineamientos de seguridad originalmente ideados en Brasil y alentados por la OPS/OMS en Latinoamérica. ${ }^{2}$ Honduras no es la excepción y con experiencias latinoamericanas que reportan eficiencia de $90 \%$ en la localización y derivación de seropositivos circulantes en la población

Cuadro 2. Seguimiento telefónico de pacientes, donadores seropositivos por Enfermedad de Chagas, Banco de Sangre, Hospital Escuela, Tegucigalpa, 2016, $n=15$.

\begin{tabular}{lcc}
\hline Seguimiento telefónico & N & (\%) \\
\hline Tipo de donante & & \\
Familiar o allegado & 13 & $(86.7)$ \\
Voluntario no remunerado & 2 & $(13.3)$ \\
Procedencia & & \\
Francisco Morazán & 8 & $(53.2)$ \\
El Paraíso & 3 & $(20.0)$ \\
Olancho & 1 & $(6.7)$ \\
Cortés & 1 & $(6.7)$ \\
Comayagua & 1 & $(6.7)$ \\
La Paz & 1 & $(6.7)$ \\
Donación de sangre en los últimos 3 años & & \\
Si & 12 & $(80.0)$ \\
No & 3 & $(20.0)$ \\
Fue informado sobre el resultado serológico & & \\
Si & 6 & $(40.0)$ \\
No & 9 & $(60.0)$ \\
\hline
\end{tabular}

general, casi interrumpiendo esa vía de transmisión y mantenimiento de endemias urbanas como en el caso de la Enfermedad de Chagas. Se recomienda la implementación de sistemas de notificación vía telefonía móvil o vía internet a donadores seropositivos, con la aplicación de algoritmos de seguimiento, localización y referencia integrados con las autoridades sanitarias dentro del marco de ley apropiado. ${ }^{12}$ Esto podría contribuir de forma importante a la eliminación de endemias urbanas 0 urbano-marginales mantenidas por individuos seropositivos circulando en la población general en condiciones de pobreza, tal como es el caso de la muestra estudiada, en donde sólo dos quintas partes fueron notificados (Cuadro 2). Es decir, que el resto circula en la población general, contribuyendo posiblemente al mantenimiento urbano de la endemia por Enfermedad de Chagas en nuestro país.

Otra problemática que aún no se ha estudiado en Honduras es el de la doble seropositividad, desconociéndose aún la importancia que podría tener a nivel local, ya que se ha descrito por ejemplo en Colombia la existencia de más de cien mil individuos con seropositividad doble por T. cruzi y hepatitis. ${ }^{21} \mathrm{Se}$ desconoce el impacto de esta situación en la salud pública a nivel nacional por lo que se recomienda realizar una investigación alrededor de esta problemática.

Entre las limitaciones de este estudio están que el muestreo utilizado, aunque fue aleatorio, fue realizado a partir de una muestra pre-seleccionada por su seropositividad; además, solo fue posible contactar al $50 \%$ de los donadores identificados. Los resultados en todo caso muestran que a través del banco de sangre se continúan detectando donadores seropositivos por $T$. cruzi y que es una oportunidad para su detección y manejo oportuno y adecuado. Fundamentados en los resultados de este estudio, se recomienda implementar un sistema digital de notificación, localización y referencia de bajo costo, para mejorar la seguridad transfusional.

\section{AGRADECIMIENTOS}

Se agradece al personal del Banco de Sangre del Hospital Escuela por el apoyo y colaboración para acceder a los registros de los donadores. Se reconoce y agradece la colaboración brindada por los donadores ya que sin su apoyo no hubiese sido posible completar este estudio.

\section{REFERENCIAS}

1. Pinto J, Brener S. Chagas disease and blood transfusion. Mem Inst Oswaldo Cruz. 1984;79:139-47.

2. Moraes-Souza H. Chagas infection transmission control: situation of transfusional transmission in Brazil and other countries of Latin America. Mem Inst Oswaldo Cruz. 1999;94 Suppl 1:419-23.

3. Moncayo A, Silveira AC. Current epidemiological trends for Chagas disease in Latin America and future challenges in epidemiology, surveillance and health policy. Mem Inst Oswaldo Cruz. 2009;104 Suppl 1:17-30.

4. Martinez-Tovar JG, Rebollar-Tellez EA, Fernandez Salas I. Seroprevalence of T. cruzi infection in blood donors and Chagas cardiomyopathy in patients from the coal mining region of Coahuila, Mexico. Rev Instit Med Trop Sao Paulo. 2014;56(2):169-74. Epub 2014/03/15.

5. Luquetti AO, Espinoza B, Martinez I, Hernandez-Becerril N, Ponce C, Ponce $\mathrm{E}$, et al. Performance levels of four Latin American laboratories for the serodiagnosis of Chagas disease in Mexican sera samples. Mem Inst Oswaldo Cruz. 2009;104(5):797-800.

6. Eder A. Transfusion Medicine-Blood Donor Selection, Testing, and Collection. In: Caplan MJ, editor. Reference module in biomedical research. Rockville, MD, USA: Elsevier/American Red Cross Biomedical Services; 2014. p. 1-11.

7. Jersild C, Hafner V. Blood Transfusion Services. In: Murray C HK, Wuah S, editor. International Encyclopedia of Public Health. Amsterdam, Holland: Academic Press; 2008. p. 325-9.

8. Zaidi N. Transfusion-related infections. In: Cohen J, Powderly W, Opal S, editors. Infectious Diseases. 3rd ed. London, UK: Mosby; 2010. p. 775-9.

9. Zia M, Taha H, Ricci C. Transfusion-Transmitted Diseases. Medscape Reference Drugs, Disease \& Procedures [Internet]. 2014[consul- 
tado abril 2016];1-11. Disponible en: http://emedicine.medscape.com/ article/1389957-overview.

10. Shaz BH. Transfusion Transmitted Diseases. In: Hillyer C, Shaz B, Zimring $\mathrm{J}$, Abshire T, editors. Transfusion Medicine and Hemostasis. Burlington MA, USA: Academic Press; 2013. p. 361-71.

11. Giraldo E, Morales M, Maya M, Rendón L, Cardona J. Prevalencia de marcadores de infecciones transmisibles y su relación con variables demográficas en un banco de sangre de Antioquía Colombia, 2010-2013. CES Med. 2015;29(1):59-73.

12. Avilés S. Seguimiento epidemiológico y experiencia del Banco Central de Sangre Centro Médico Nacional "La Raza". Rev Mex Med Trans. 2011;4(2):62-5.

13. Secretaría de Salud (HN). Plan estratégico para la prevención, atención, control y eliminación de enfermedades infecciosas desatendidas en Honduras (PEEDH) 2012-2017. Tegucigalpa: SESAL; 2012.

14. Ponce $C$, Zeledón R. La enfermedad de Chagas en Honduras. Bol Oficina Sanit Panam. 1973;75(3):239-48

15. Secretaría de Salud $(\mathrm{HN})$, Dirección General de Promoción de la Salud, Programa Nacional de Prevención y Control de la Enfermedad Chagas.
Agencia de Cooperación Internacional (JICA). Proyecto de Control de la Enfermedad de Chagas Fase 2 (2008-2011). Informe Final. Tegucigalpa, M.D.C., Honduras, C.A.: SESAL, JICA; 2011.

16. Instituto de Enfermedades Infecciosas y Parasitología Antonio Vidal. Manual de manejo de enfermedades parasitarias prioritarias en Honduras. $2^{\text {a }}$ ed. Tegucigalpa: OPS; 2009.

17. Organización Panamericana de la Salud, Organización Mundial de la Salud. Salud en las Américas, Panorama regional perfiles de país: Honduras. Washington, D.C.: OPS, OMS; 2012.

18. Anteproyecto para la Creación del Banco Nacional de Sangre. Rev Méd Hondur. 1981;49(2):68-71.

19. Honduras. Congreso Nacional. Reglamento de la Ley Especial sobre VIH/ SIDA. Acuerdo Ejecutivo No. 0009. Diario Oficial La Gaceta, 25 de Julio de 2003.

20. Honduras. Congreso Nacional. Funciones y atribuciones del Consejo Nacional de la Sangre. Diario Oficial La Gaceta, 31 de Mayo de 1997.

21. Beltrán M, Forero M, Ayala M, Vélez R. Los bancos de sangre en la vigilancia en Salud Pública de enfermedades transmitidas por la sangre. Inf Quinc Epidemiol Nac. 2002;7(4):54-60.

ABSTRACT. Background: The elimination of non-vector transmission of Trypanosoma cruzi is a national objective of public health. Seroprevalence in blood donors is target for elimination strategies for Chagas Disease. Objective: To characterize seropositive donors by T. cruzi and their management in the Blood Bank, Hospital Escuela, Tegucigalpa, 2016. Methodology: Cross-sectional descriptive study on a random sample of 150 blood donors, over 18 years old, selected from a sub-group of 726 seropositive by mandatory reporting diseases. The T. cruzi seropositive donors were invited to participate by telephone follow-up. Information on diagnosis, sociodemographic characteristics, notification, reference and follow-up was recorded. The protocol was approved by Facultad de Ciencias Médicas UNAH institutional review board. Results: Thirty T. cruzi seropositive cases were identified $(20.0 \%, 95 \% \mathrm{Cl} 13.3-$ 26.7), average age 39.1 years, male $66.7 \%$ (20), coming from the center-south-east regions and low socioeconomic status. Of these 30 cases, 15 responded and agreed to participate; of these, 6 reported that they were notified about the serological result and 5 were referred for evaluation and treatment. Among these 5 , in one case a second diagnostic test was performed. None was notified about their ability to donate blood. Conclusion: The cases identified are young adults, with a man to woman ratio of $2: 1$. It is necessary to implement a national registry system for notification and follow-up of the cases identified in blood banks. This system must be integrated into the surveillance system of the Honduras Secretary of Health.

Keywords: Blood component transfusion, Chagas Disease, Transfusion medicine, Trypanosoma cruzi. 